Supporting Information

\title{
Revolutionizing Membrane Design Using Machine Learning-Bayesian Optimization
}

Haiping Gao ${ }^{\mathrm{a}, \#}$, Shifa Zhonga,\#, Wenlong Zhang ${ }^{\mathrm{a}}$, Thomas Igou ${ }^{\mathrm{a}}$, Eli Berger ${ }^{\mathrm{a}}$, Elliot Reid ${ }^{\mathrm{a}}$, Yangying Zhao ${ }^{\mathrm{a}}$, Dylan Lambeth ${ }^{\mathrm{a}}$, Lan Gan ${ }^{\mathrm{a}}$, Moyosore A. Afolabi ${ }^{\mathrm{a}}$, Zhaohui Tong ${ }^{\mathrm{b}}$, Guanghui Lan $^{\mathrm{c}}$, Yongsheng Chen ${ }^{\mathrm{a}, *}$

a School of Civil and Environmental Engineering, Georgia Institute of Technology, Atlanta, Georgia 30332, United States

b Agriculture and Biological Engineering, Institute of Food and Agricultural Sciences, University of Florida, Gainesville, Florida 32611, United States

${ }^{\mathbf{c}} \mathrm{H}$. Milton Stewart School of Industrial and Systems Engineering, Georgia Institute of Technology, Atlanta, Georgia 30332, United States

\# These authors contributed equally to this work.

* Corresponding author: Yongsheng Chen, Email: yongsheng.chen@ce.gatech.edu, Phone: 4048943089

\section{Supporting information includes 14 pages with the following texts, figures, and tables as cross referenced throughout the main article:}

Text S1: Four descriptors to represent chemicals

Text S2: Brief introduction of Morgan fingerprint

Text S3 Brief introduction of molecular fingerprint overlapping

Figure S1: Four representations for piperazine (PIP).

Figure S2: Decomposing the Morgan fingerprint of PIP to their representative atoms or groups.

Figure S3: An example of fingerprint overlapping.

Figure S4: The SHAP plot of features in the Morgan fingerprint.

Figure S5: Group 2 monomers used to perform Bayesian fabrication optimization.

Table S1: Description of input features for model development.

Table S2: Description of key characteristics for salt ions.

Table S3: Statistical information of the numerical features as model inputs.

Table S4: Screening candidates for Encoder and Scaler methods.

Table S5: Materials and fabrication conditions used for membrane fabrication to validate prediction accuracy of the built ML models.

Table S6: Monomers and fabrication conditions used to fabricate membranes for validation of Bayesian optimization on water permeability and water/ $/ \mathrm{Na}_{2} \mathrm{SO}_{4}$ selectivity.

Table S7: Monomers and fabrication conditions used to fabricate membranes for validation of Bayesian optimization on water permeability and water/ $\mathrm{NaCl}$ selectivity.

Table S8: Predictive performance of different configurations of machine learning algorithms, encoder methods and scaler methods. 
Text S1 Four descriptors to represent chemicals

Four descriptors for a chemical include: molecular descriptor (MD), molecular fingerprint (MF), molecular image (MI) and molecular graph (MG). MD represents a chemical by its physicochemical properties, such as molecular weight, volume, and charge. MF encode a chemical into a binary vector (only containing $0 \mathrm{~s}$ and $1 \mathrm{~s}$ ), in which only $1 \mathrm{~s}$ represent certain atom groups (Text S1). MI represent a chemical by a 2D image, which can be handled by convolution neural network. MG treats a chemical as a graph, in which nodes are the atoms while the edge are the bonds. MG can be handled by graph neural network. These four representations were illustrated in Figure S1. Molecular fingerprint holds a distinct advantage over other descriptors, where not only the number of each atom type in a repeat unit is included but also the chemical connectivity between different units is considered. Moreover, molecular fingerprint is an inherently more dynamic descriptive method since it evolves to include materials as they are synthesized.
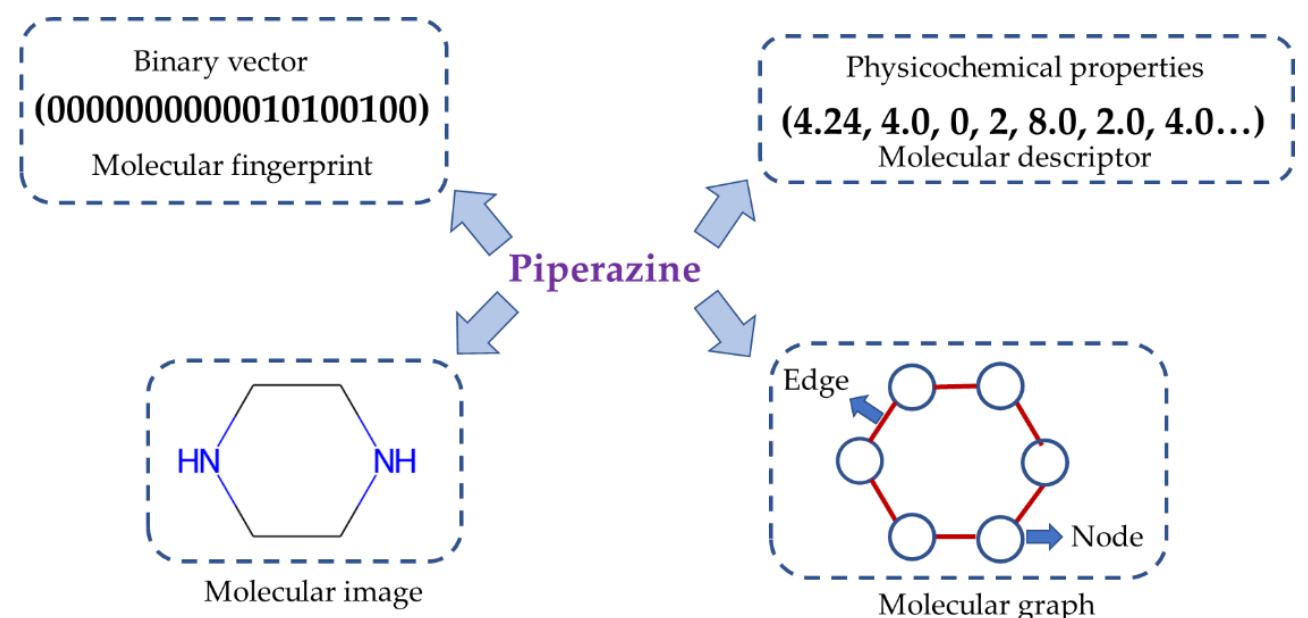

Piperazine

Figure S1. Four representations for piperazine (PIP).

Text S2 Brief introduction of Morgan fingerprint

Here, we illustrated the concepts of Morgan fingerprint by taking PIP as an example. As shown in Figure S2, the Morgan fingerprint of PIP have length of 2433 and radius of 1, which is a binary vector. There are only $0 \mathrm{~s}$ and $1 \mathrm{~s}$ in the vector, in which only $1 \mathrm{~s}$ represent that there are atom groups there. The position of $1 \mathrm{~s}$ represents different types of atom groups. We can also check what the specific atom groups represented by $1 \mathrm{~s}$, as shown in Figure S2. The PIP is decomposed into four atom groups and each group is stored in a different position in the vector with 1 filled in. This is how Morgan fingerprint represents a specific chemical. 


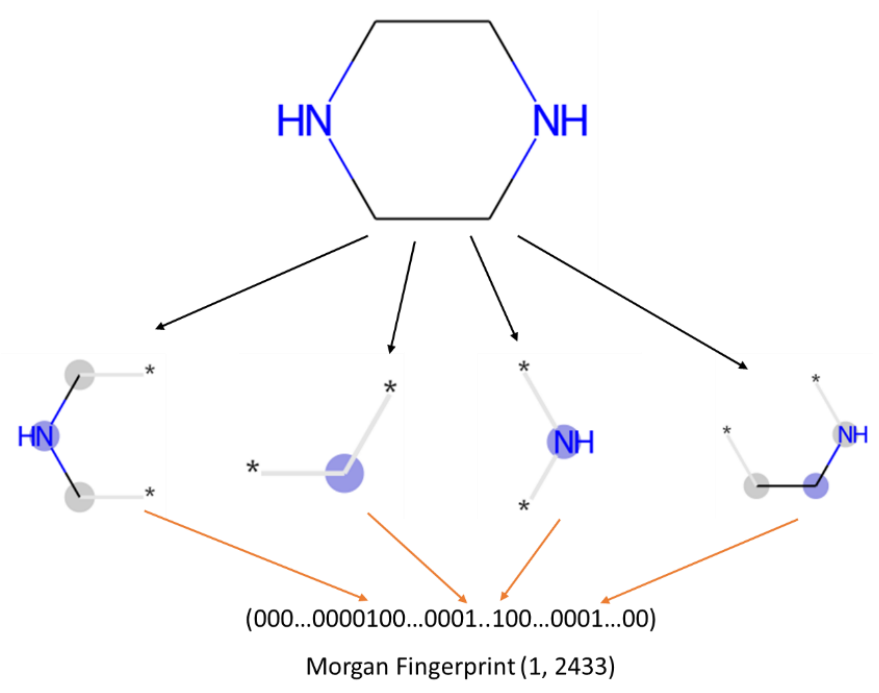

Figure S2. Decomposing the Morgan fingerprint of PIP to their representative atoms or groups.

Text S3 Brief introduction of molecular fingerprint overlapping

There are situations where more than one fingerprint overlaps each other. The following example in Figure S3 shows the fingerprint overlapping when the bit position was set to 1. Notably, two different groups, amine group and sulfonic group were encoded into the same position in the Morgan fingerprint.
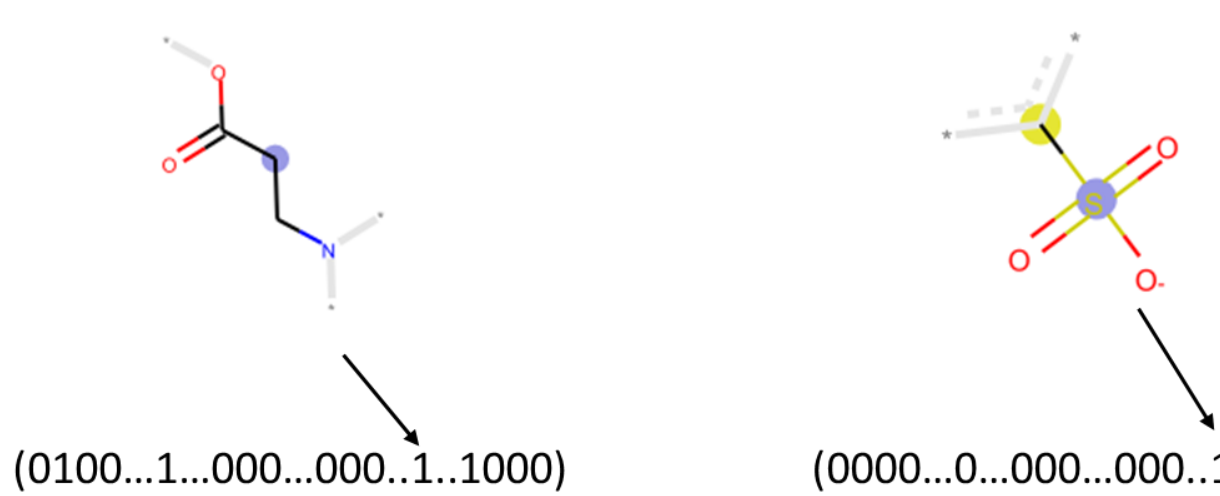

(0100...1...000...000..1..1000)

$(0000 \ldots 0 . . .000 \ldots 000 . .1 . .0001)$

Figure S3. An example of fingerprint overlapping. 

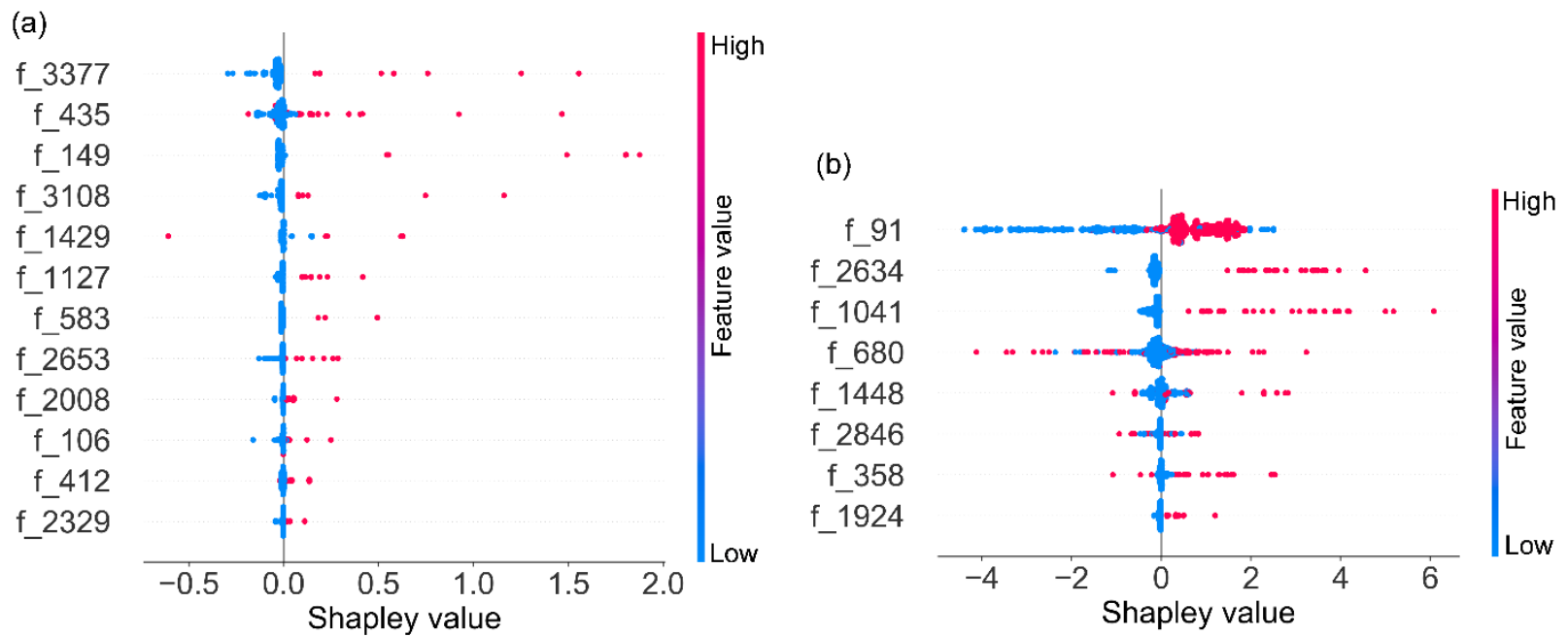

Figure S4. The SHAP plot of features in the Morgan fingerprint. (a), beneficial features for water permeability in the training dataset. (b), beneficial features for salt rejection in the training dataset. The $\mathrm{X}$-axes are the Shapley values where the positive value means that the water permeability and the salt rejection can be increased by the specific features listed in both (a) and (b), while the negative value indicates a resultant reduction in water permeability and salt rejection. The size of each feature's value is colored from blue to red, corresponding to the smallest and largest values. The pattern for each feature is composed of small dots and each dot represents one sample containing this feature.<smiles>CC(N)CN</smiles>

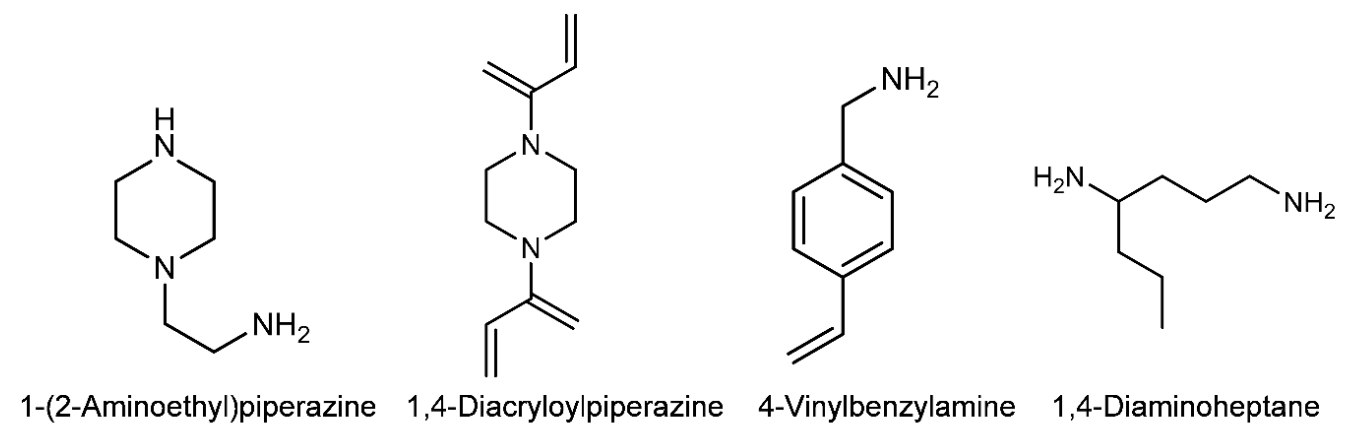

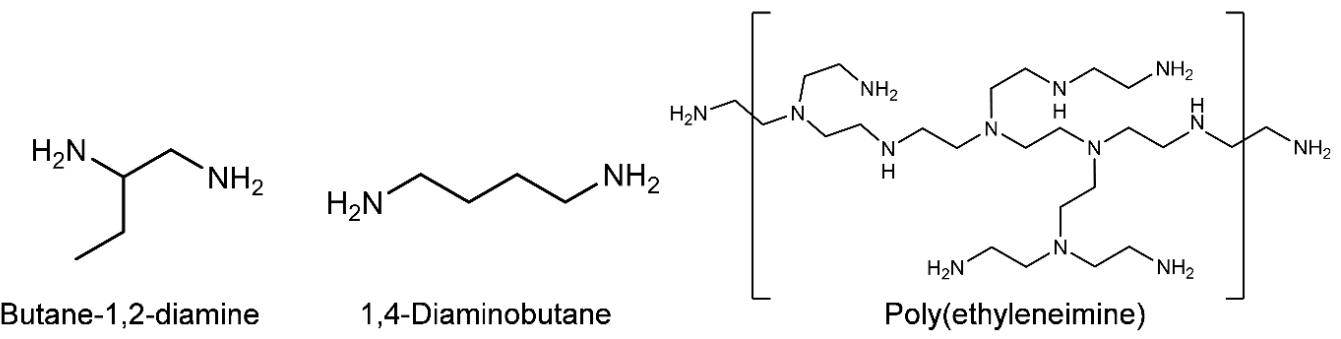

Figure S5. Group 2 monomers used to perform Bayesian fabrication optimization. 
Table S1. Description of input features for model development.

\begin{tabular}{|c|c|c|c|}
\hline Category & Feature & Unit & Description \\
\hline \multirow{5}{*}{$\begin{array}{l}\text { Monomer } \\
\text { selection }\end{array}$} & monomer A1 & I & The first monomer used in aqueous phase \\
\hline & monomer A2 & / & The second monomer used in aqueous phase \\
\hline & $a-m w$ & $\mathrm{~g} \mathrm{~mol}^{-1}$ & The molecular weight of the first monomer \\
\hline & b-mw & $\mathrm{g} \mathrm{mol}^{-1}$ & The molecular weight of the second monomer \\
\hline & monomer B & l & The monomer used in organic phase \\
\hline \multirow{18}{*}{$\begin{array}{l}\text { Fabrication } \\
\text { condition }\end{array}$} & A2/A1 ratio & / & $\begin{array}{l}\text { The weight fraction ratio of two monomers used in } \\
\text { aqueous phase solution }\end{array}$ \\
\hline & $\mathrm{C}$ & $\mathrm{wt} \%$ & $\begin{array}{l}\text { The weight fraction of monomer }(\mathrm{A} 1+\mathrm{A} 2) \text { in } \\
\text { aqueous phase }\end{array}$ \\
\hline & $\mathrm{C}(\mathrm{B})$ & $\mathrm{wt} \%$ & The weight fraction of monomer in organic phase \\
\hline & organic solvent type & l & $\begin{array}{l}\text { The organic solvent used to prepare organic phase } \\
\text { solution }\end{array}$ \\
\hline & additive $\mathrm{X} 1$ type-aqueous & l & The first additive used in the aqueous phase \\
\hline & additive $\mathrm{X} 2$ type-aqueous & l & The second additive used in aqueous phase solution \\
\hline & $\mathrm{C}$ (additive $\mathrm{X} 1$ ) & $\mathrm{wt} \%$ & $\begin{array}{l}\text { The weight fraction of the first additive in aqueous } \\
\text { phase }\end{array}$ \\
\hline & $\mathrm{C}$ (additive $\mathrm{X} 2$ ) & $\mathrm{wt} \%$ & $\begin{array}{l}\text { The weight fraction of the second additive in } \\
\text { aqueous phase }\end{array}$ \\
\hline & $\mathrm{pH}$ & I & The $\mathrm{pH}$ value of aqueous phase solution \\
\hline & additive $\mathrm{Y}$ & / & The additive used in organic phase solution \\
\hline & $\mathrm{C}(\mathrm{Y})$ & $\mathrm{wt} \%$ & $\begin{array}{l}\text { The weight fraction of additive used in organic } \\
\text { phase solution }\end{array}$ \\
\hline & $\begin{array}{l}\text { nanomaterials type in } \\
\text { aqueous phase }\end{array}$ & & The nanomaterials added into the aqueous phase. \\
\hline & $\begin{array}{l}\text { nanomaterials type in } \\
\text { organic phase }\end{array}$ & & The nanomaterials added into the organic phase. \\
\hline & $\mathrm{C}$ (nanomaterials-aqueous) & $\mathrm{wt} \%$ & $\begin{array}{l}\text { The weight fraction of nanomaterials in aqueous } \\
\text { phase solution }\end{array}$ \\
\hline & $\mathrm{C}$ (nanomaterials-organic) & $\mathrm{wt} \%$ & $\begin{array}{l}\text { The weight fraction of nanomaterials in organic } \\
\text { phase solution }\end{array}$ \\
\hline & nanomaterials morphology & l & The shape of nanomaterials \\
\hline & $\mathrm{t}$ (polymerization) & $\mathrm{s}$ & The time used for interfacial polymerization \\
\hline & $\mathrm{t}$ (heat curing) & $\min$ & The heat-curing time used for further cross-linking \\
\hline
\end{tabular}




\begin{tabular}{|c|c|c|c|}
\hline & $\mathrm{T}$ (heat curing) & ${ }^{\circ} \mathrm{C}$ & $\begin{array}{l}\text { The heat-curing temperature for further cross- } \\
\text { linking }\end{array}$ \\
\hline & substrate type & / & $\begin{array}{l}\text { The type of substrate membrane for interfacial } \\
\text { polymerization }\end{array}$ \\
\hline & $\mathrm{r}$ (substrate) & $\mathrm{kDa}$ & The molecular weight cutoff of the substrate \\
\hline \multirow{7}{*}{$\begin{array}{l}\text { Operation } \\
\text { setting }\end{array}$} & TMP & bar & $\begin{array}{c}\text { The transmembrane pressure applied for membrane } \\
\text { tests. }\end{array}$ \\
\hline & $\mathrm{C}$ (Salt) & $\mathrm{mg} \mathrm{L}^{-1}$ & The concentration of salt used for salt rejection tests \\
\hline & valence & & The valence of salt ions \\
\hline & ionic radius & $\AA$ & The ionic radius of salt ions \\
\hline & hydrated radius & $\AA$ & The Stokes radius of salt ions \\
\hline & Stokes radius & $\AA$ & The hydrated radius of salt ions \\
\hline & hydration free energy & $\mathrm{kJ} \mathrm{mol}^{-1}$ & The hydration free energy of salt ions \\
\hline \multirow{2}{*}{$\begin{array}{l}\text { Membrane } \\
\text { performance }\end{array}$} & water permeability & $\mathrm{Lm}^{-2} \mathrm{~h}^{-1} \mathrm{bar}^{-1}$ & $\begin{array}{l}\text { The pure water permeability of the tested } \\
\text { membranes }\end{array}$ \\
\hline & salt rejection & $\%$ & The salt rejection of the tested membranes \\
\hline
\end{tabular}

Table S2. Description of key characteristics for salt ions.

\begin{tabular}{|c|c|c|c|c|c|c|}
\hline Ions & Valence & $\begin{array}{c}\text { Ionic } \\
\text { radius }^{\text {a }} \\
\AA\end{array}$ & $\begin{array}{c}\text { Hydrated } \\
\text { radius }^{\text {a }} \\
\AA\end{array}$ & $\begin{array}{c}\text { Stokes } \\
\text { radius }^{\text {a }} \\
\AA\end{array}$ & $\begin{array}{c}\text { Hydration free } \\
\text { energy }^{b} \\
\text { kcal mol }^{-1}\end{array}$ & Salt used \\
\hline \multicolumn{7}{|l|}{ Anions } \\
\hline $\mathrm{Cl}^{-}$ & -1 & 1.81 & 3.32 & 1.21 & 340 & $\mathrm{NaCl}$ \\
\hline $\mathrm{SO}_{4}{ }^{2-}$ & -2 & 2.90 & 3.82 & 2.90 & 1080 & $\mathrm{Na}_{2} \mathrm{SO}_{4}$ \\
\hline $\mathrm{NO}_{3}^{-}$ & -1 & & & & & $\mathrm{NaNO}_{3}$ \\
\hline \multicolumn{7}{|l|}{ Cations } \\
\hline $\mathrm{Na}^{+}$ & +1 & 0.95 & 3.58 & 1.84 & 365 & $\mathrm{NaCl}$ \\
\hline $\mathrm{Mg}^{2+}$ & +2 & 0.65 & 4.23 & 3.47 & 1830 & $\mathrm{MgCl}_{2} / \mathrm{MgSO}_{4}$ \\
\hline $\mathrm{Ca}^{2+}$ & +2 & 0.99 & 4.12 & 3.10 & 1505 & $\mathrm{CaCl}_{2}$ \\
\hline $\mathrm{Li}^{+}$ & +1 & & & & & $\mathrm{LiCl}$ \\
\hline
\end{tabular}

${ }^{\mathrm{a}}$ Data from Liang, Y. (2020) ${ }^{1} ;{ }^{\mathrm{b}}$ Data from Marcus, Y. $(1991)^{2}$ 
Table S3. Statistical information of the numerical features as model inputs.

\begin{tabular}{|c|c|c|c|c|c|c|c|c|}
\hline Feature & Count & Mean & std & Min & $25 \%$ & $50 \%$ & $75 \%$ & Max \\
\hline \multicolumn{9}{|c|}{$\underline{\text { A dataset }}$} \\
\hline $\mathrm{A} 2 / \mathrm{A} 1$ ratio & 567 & 0.119282 & 0.623658 & 0 & 0 & 0 & 0 & 9.091 \\
\hline $\mathrm{C}(\mathrm{A})$ & 567 & 1.3102116 & 1.1576797 & 0.03 & 0.35 & 1 & 2 & 7 \\
\hline $\mathrm{C}(\mathrm{B})$ & 567 & 0.2273942 & 0.1641726 & 0.015 & 0.15 & 0.2 & 0.285 & 1.2 \\
\hline $\mathrm{C}$ (additive $\mathrm{X} 1$ ) & 528 & 0.498534 & 2.482685 & 0 & 0 & 0 & 0.1 & 40 \\
\hline $\mathrm{C}$ (additive $\mathrm{X} 2$ ) & 528 & 0.203655 & 0.887028 & 0 & 0 & 0 & 0 & 10 \\
\hline $\mathrm{pH}$ & 133 & 10.4812 & 1.615978 & 10 & 10 & 11 & 11.5 & 12 \\
\hline $\mathrm{C}$ (additive $\mathrm{Y}$ ) & 566 & 0.016431 & 0.150775 & 0 & 0 & 0 & 0 & 2 \\
\hline $\mathrm{C}$ (nanomaterials-aqueous) & 564 & 0.030741 & 0.140144 & 0 & 0 & 0 & 0 & 1.5 \\
\hline $\mathrm{C}$ (nanomaterials-organic) & 567 & 0.021395 & 0.239772 & 0 & 0 & 0 & 0 & 4.62 \\
\hline $\mathrm{t}$ (polymerization) & 567 & 127.3898 & 264.0877 & 40 & 40 & 60 & 120 & 1800 \\
\hline $\mathrm{t}$ (heat curing) & 472 & 11.68538 & 10.52411 & 5 & 5 & 10 & 15 & 120 \\
\hline $\mathrm{T}$ (heat curing) & 513 & 63.74464 & 17.38205 & 50 & 50 & 60 & 80 & 100 \\
\hline r (substrate) & 337 & 50.17804 & 47.52472 & 20 & 20 & 50 & 50 & 500 \\
\hline TMP & 567 & 5.88496 & 2.45254 & 4 & 4 & 6 & 6 & 17.24 \\
\hline water permeability & 567 & 8.844782 & 5.836301 & 4.65 & 4.65 & 7.6 & 11.85429 & 34.53 \\
\hline \multicolumn{9}{|c|}{$\underline{\mathbf{R} \text { dataset }}$} \\
\hline $\mathrm{A} 2 / \mathrm{A} 1$ ratio & 1524 & 0.115972 & 0.633935 & 0 & 0 & 0 & 0 & 9.091 \\
\hline $\mathrm{C}(\mathrm{A})$ & 1524 & 1.294928 & 0.975413 & 0.03 & 0.5 & 1 & 2 & 7 \\
\hline $\mathrm{C}(\mathrm{B})$ & 1524 & 0.222211 & 0.163285 & 0.015 & 0.15 & 0.15 & 0.25 & 1.2 \\
\hline $\mathrm{C}$ (additive $\mathrm{X} 1$ ) & 1437 & 0.392494 & 1.63153 & 0 & 0 & 0 & 0 & 4 \\
\hline $\mathrm{C}$ (additive $\mathrm{X} 2$ ) & 1395 & 0.195355 & 0.892006 & 0 & 0 & 0 & 0 & 10 \\
\hline $\mathrm{pH}$ & 327 & 10.56422 & 1.615826 & 7 & 10 & 11.5 & 11.5 & 12 \\
\hline $\mathrm{C}$ (additive $\mathrm{Y}$ ) & 1520 & 0.017664 & 0.15868 & 0 & 0 & 0 & 0 & 2 \\
\hline $\mathrm{C}$ (nanomaterials-aqueous) & 1515 & 0.027781 & 0.114373 & 0 & 0 & 0 & 0.0001 & 1.5 \\
\hline $\mathrm{C}$ (nanomaterials-organic) & 1524 & 0.028835 & 0.290222 & 0 & 0 & 0 & 0 & 4.62 \\
\hline $\mathrm{t}$ (polymerization) & 1524 & 116.0925 & 256.1591 & 5 & 30 & 60 & 120 & 1800 \\
\hline $\mathrm{t}$ (heat curing) & 1213 & 12.0474 & 11.12517 & 0 & 5 & 10 & 15 & 120 \\
\hline $\mathrm{T}$ (heat curing) & 1292 & 62.21672 & 16.921 & 22 & 50 & 60 & 70 & 100 \\
\hline $\mathrm{r}$ (substrate) & 845 & 54.63905 & 54.47743 & 10 & 20 & 50 & 50 & 500 \\
\hline TMP & 1524 & 5.675026 & 2.412454 & 1.5 & 4 & 5 & 6 & 17.24 \\
\hline salt concentration & 1524 & 1294.262 & 542.9373 & 116.9 & 1000 & 1000 & 2000 & 3000 \\
\hline salt rejection & 1524 & 67.55691 & 29.11877 & 13 & 40 & 78 & 95 & 100 \\
\hline
\end{tabular}


Table S4. Screening candidates for Encoder and scaler methods.

\begin{tabular}{|c|c|c|}
\hline Methods & Candidates & Working principle \\
\hline \multirow{8}{*}{$\begin{array}{l}\text { Encoding } \\
\text { methods }\end{array}$} & BackwardDifferenceEncoder & $\begin{array}{l}\text { The mean of the dependent variable for a level is compared with the } \\
\text { mean of the dependent variable for the prior level. }\end{array}$ \\
\hline & MEstimateEncoder, & $\begin{array}{l}\text { M-Estimate Encoder is a simplified version of Target Encoder. It has } \\
\text { only one hyper-parameter }-\mathrm{m} \text {, which represents the power of } \\
\text { regularization. }\end{array}$ \\
\hline & SumEncoder & $\begin{array}{l}\text { Sum Encoder compares the mean of the dependent variable (target) } \\
\text { for a given level of a categorical column to the overall mean of the } \\
\text { target }\end{array}$ \\
\hline & BinaryEncoder & $\begin{array}{l}\text { Converting a category into binary digits. If there are } n \text { unique } \\
\text { categories, then binary encoding results in the only } \log (\text { base } 2)^{\mathrm{n}} \\
\text { features }\end{array}$ \\
\hline & JamesSteinEncoder & $\begin{array}{l}\text { For feature value, the James-Stein estimator returns a weighted } \\
\text { average of: The mean target value for the observed feature value or } \\
\text { the mean target value (regardless of the feature value). }\end{array}$ \\
\hline & OneHotEncoder & Mapping each category to a vector that contains 0 and 1 . \\
\hline & BaseNEncoder & $\begin{array}{l}\text { Base- } \mathrm{N} \text { encoder encodes the categories into arrays of their base- } \mathrm{N} \\
\text { representation. A base of } 1 \text { is equivalent to one-hot encoding (not } \\
\text { really base- } 1 \text {, but useful), a base of } 2 \text { is equivalent to binary } \\
\text { encoding. } \mathrm{N}=\text { number of actual categories is equivalent to vanilla } \\
\text { ordinal encoding. }\end{array}$ \\
\hline & HelmertEncoder & $\begin{array}{l}\text { The mean of the dependent variable for a level is compared to the } \\
\text { mean of the dependent variable over all previous levels }\end{array}$ \\
\hline \multirow{5}{*}{$\begin{array}{l}\text { Scaler } \\
\text { methods }\end{array}$} & StandardScaler & $\begin{array}{l}\text { Standardize features by removing the mean and scaling to unit } \\
\text { variance }\end{array}$ \\
\hline & MinMaxScaler & $\begin{array}{l}\text { Transform features by scaling each feature to a given range. Here, it } \\
\text { is }[0,1] \text {. }\end{array}$ \\
\hline & MaxAbsScaler & Scale each feature by its maximum absolute value. \\
\hline & RoubustScaler & Scale features using statistics that are robust to outliers \\
\hline & Powertransform & $\begin{array}{l}\text { Apply a power transform feature-wise to make data more Gaussian- } \\
\text { like. }\end{array}$ \\
\hline
\end{tabular}

Table S5. Materials and fabrication conditions used for membrane fabrication to validate prediction accuracy of the built ML models.

\begin{tabular}{|c|c|c|c|c|}
\hline \multirow{2}{*}{$\begin{array}{c}\text { Material and } \\
\text { fabrication } \\
\text { condition }\end{array}$} & \multicolumn{4}{|c|}{ Membrane } \\
\hline & 1 & 2 & 3 & 4 \\
\hline $\begin{array}{l}\text { Amine material } \\
\text { (aqueous phase) }\end{array}$ & $\begin{array}{c}1,2- \\
\text { diaminopropane }\end{array}$ & 1,2-diaminopropane & 1,2-diaminopropane & $\begin{array}{l}\text { polyethylenime } \\
\text { (PEI) }\end{array}$ \\
\hline $\begin{array}{l}\text { Molecular } \\
\text { structure }\end{array}$ & ${ }_{\mathrm{NH}_{2}}^{\mathrm{H}_{3} \mathrm{C}} \underbrace{\mathrm{CH}_{2} \mathrm{NH}_{2}}_{\mathrm{CH}_{2}}$ & $\mathrm{H}_{3} \mathrm{C}{\underset{N H_{2}}{\mathrm{CH}_{2} \mathrm{NH}_{2}}}^{\mathrm{N}_{2}}$ & 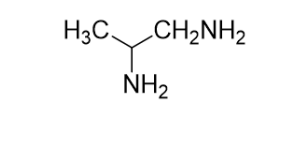 & {$\left[\begin{array}{ll}n \\
{[}\end{array}\right]_{n}$} \\
\hline $\begin{array}{l}\text { Molecular } \\
\text { Weight } \\
\left(\mathrm{g} \mathrm{mol}^{-1}\right)\end{array}$ & 74.125 & 74.125 & 74.125 & 1300 \\
\hline
\end{tabular}




\begin{tabular}{|c|c|c|c|c|}
\hline $\begin{array}{l}\text { C(A), } \\
\text { wt \% }\end{array}$ & 0.25 & 0.15 & 0.15 & 0.50 \\
\hline Additive X1 & l & $\begin{array}{l}\text { sodium dodecyl } \\
\text { sulfate }\end{array}$ & $\begin{array}{l}\text { sodium } \\
\text { carbonate }\end{array}$ & l \\
\hline $\begin{array}{c}\mathrm{C} \text { (additive } \mathrm{X} 1) \text {, } \\
\text { wt } \%\end{array}$ & I & 0.05 & 0.10 & l \\
\hline $\begin{array}{l}\text { Organic phase } \\
\text { material }\end{array}$ & $\begin{array}{l}\text { trimesoyl chloride } \\
\text { (TMC) }\end{array}$ & TMC & TMC & TMC \\
\hline $\begin{array}{l}\mathrm{C}(\mathrm{B}) \\
\text { wt } \%\end{array}$ & 0.3 & 0.225 & 0.225 & 0.5 \\
\hline $\begin{array}{l}\text { Organic } \\
\text { solvent }\end{array}$ & n-Hexane & n-Hexane & n-Hexane & n-Hexane \\
\hline $\begin{array}{l}\text { Polymerization } \\
\text { time (s) }\end{array}$ & 60 & 60 & 60 & 60 \\
\hline $\begin{array}{c}\text { Heat curing } \\
\text { temperature }\left({ }^{\circ} \mathrm{C}\right)\end{array}$ & 60 & 60 & 60 & 60 \\
\hline $\begin{array}{l}\text { Heat curing time } \\
(\min )\end{array}$ & 10 & 10 & 10 & 10 \\
\hline $\begin{array}{c}\text { Substrate } \\
\text { type }\end{array}$ & PES & PES & PES & PES \\
\hline $\begin{array}{c}\text { Substrate } \\
\text { pore size }(\mu m)\end{array}$ & 0.2 & 0.2 & 0.2 & 0.2 \\
\hline $\begin{array}{l}\text { Transmembrane } \\
\text { pressure (TMP, bar) }\end{array}$ & 5 & 5 & 5 & 5 \\
\hline $\begin{array}{c}\text { Salt } \\
\text { used }\end{array}$ & $\mathrm{Na}_{2} \mathrm{SO}_{4}$ & $\mathrm{MgSO}_{4}$ & $\mathrm{MgSO}_{4}$ & $\mathrm{Na}_{2} \mathrm{SO}_{4}$ \\
\hline $\begin{array}{l}\text { Salt concentration } \\
\left(\mathrm{mg} \mathrm{L}^{-1}\right)\end{array}$ & 1000 & 1000 & 1000 & 1000 \\
\hline Material and & \multicolumn{4}{|c|}{ Membrane } \\
\hline condition & 5 & 6 & 7 & 8 \\
\hline $\begin{array}{l}\text { Amine material } \\
\text { (aqueous phase) }\end{array}$ & $\begin{array}{l}\text { polyethylenime } \\
\text { (PEI) }\end{array}$ & $\begin{array}{l}\text { polyethylenime } \\
\text { (PEI) }\end{array}$ & $\begin{array}{l}\text { piperazine } \\
\text { (PIP) }\end{array}$ & $\begin{array}{l}\text { piperazine } \\
\text { (PIP) }\end{array}$ \\
\hline $\begin{array}{l}\text { Molecular } \\
\text { structure }\end{array}$ & & & & \\
\hline Molecular & & & & \\
\hline $\begin{array}{l}\text { Weight } \\
\left(\mathrm{g} \mathrm{mol}^{-1}\right)\end{array}$ & 1300 & 1300 & 86.136 & 86.136 \\
\hline $\begin{array}{l}\mathrm{C}(\mathrm{A}) \text {, } \\
\text { wt } \%\end{array}$ & 0.15 & 0.15 & 0.25 & 0.25 \\
\hline Additive X1 & $\begin{array}{l}\text { sodium dodecyl } \\
\text { sulfate }\end{array}$ & $\begin{array}{l}\text { sodium } \\
\text { phosphate }\end{array}$ & $\begin{array}{l}\text { sodium dodecyl } \\
\text { sulfate }\end{array}$ & $\begin{array}{l}\text { sodium dodecyl } \\
\text { sulfate }\end{array}$ \\
\hline $\begin{array}{c}\mathrm{C} \text { (additive } \mathrm{X} 1) \text {, } \\
\text { wt } \%\end{array}$ & 0.05 & 0.075 & 0.06 & 0.06 \\
\hline $\begin{array}{l}\text { Organic phase } \\
\text { material }\end{array}$ & TMC & TMC & TMC & TMC \\
\hline $\begin{array}{l}\mathrm{C}(\mathrm{B}) \\
\text { wt } \%\end{array}$ & 0.225 & 0.225 & 0.30 & 0.30 \\
\hline $\begin{array}{l}\text { Organic } \\
\text { solvent }\end{array}$ & n-Hexane & n-Hexane & n-Hexane & n-Hexane \\
\hline
\end{tabular}




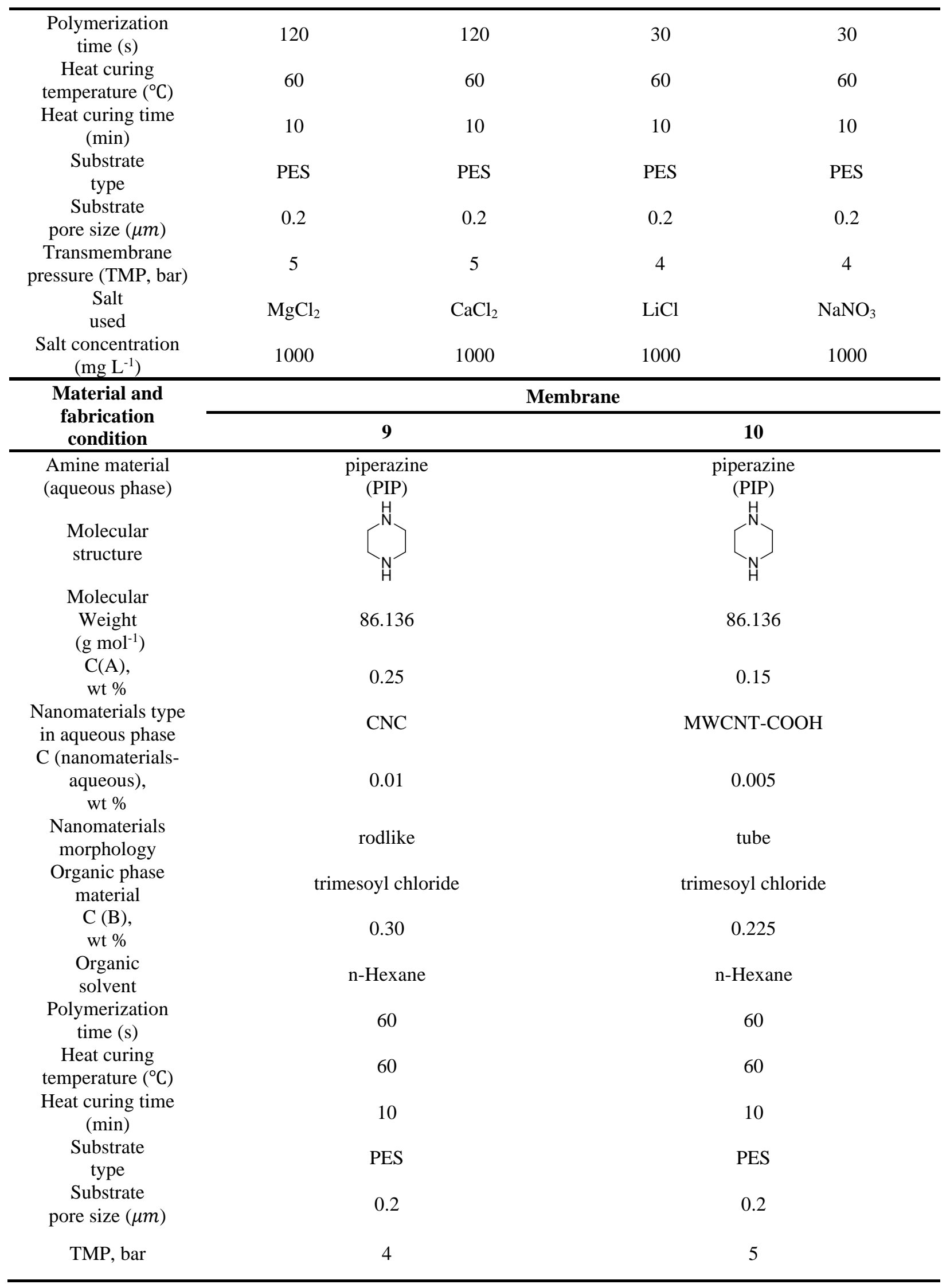




\begin{tabular}{ccc}
\hline Salt & $\mathrm{Na}_{2} \mathrm{SO}_{4}$ & $\mathrm{MgCl}_{2}$ \\
$\begin{array}{c}\text { Salt concentration } \\
\left(\mathrm{mg} \mathrm{L}^{-1}\right)\end{array}$ & 1000 & 1000 \\
\hline
\end{tabular}

Table S6. Membrane materials and fabrication conditions used to fabricate membranes for validation of Bayesian optimization on water permeability and water/ $\mathrm{Na}_{2} \mathrm{SO}_{4}$ selectivity.

\begin{tabular}{|c|c|c|c|c|}
\hline \multirow{2}{*}{$\begin{array}{l}\text { Material and fabrication } \\
\text { condition }\end{array}$} & \multicolumn{4}{|c|}{ Membrane } \\
\hline & 1 & 2 & 3 & 4 \\
\hline Material A1 & $\begin{array}{c}1-(2- \\
\text { aminoethyl)pi } \\
\text { perazine }\end{array}$ & 1,2-diaminoprone & $\begin{array}{c}2,5- \\
\text { diaminopentanoic } \\
\text { acid }\end{array}$ & $\begin{array}{c}\text { polyethylenime } \\
1300\end{array}$ \\
\hline Material A2 & $\begin{array}{c}1,2- \\
\text { diaminoprone }\end{array}$ & $\begin{array}{l}\text { tri(2-aminoethyl)- } \\
\text { amine }\end{array}$ & $\begin{array}{l}\text { polyethylenime } \\
1300\end{array}$ & $\begin{array}{c}1,4- \\
\text { diaminobutane }\end{array}$ \\
\hline $\mathrm{A} 1 / \mathrm{A} 2$ ratio & 6.6 & 0.15 & 1.7 & 0.88 \\
\hline $\mathrm{C}(\mathrm{A}), \mathrm{wt} \%$ & 0.65 & 0.77 & 0.52 & 1.25 \\
\hline Monomer B & TMC & TMC & TMC & TMC \\
\hline $\mathrm{C}(\mathrm{B}), \mathrm{wt} \%$ & 0.73 & 0.89 & 0.65 & 1.0 \\
\hline $\begin{array}{l}\text { Organic solvent } \\
\text { Additive X1 }\end{array}$ & $\begin{array}{l}\text { n-Hexane } \\
\text { sodium } \\
\text { phosphate }\end{array}$ & $\begin{array}{c}\text { n-Hexane } \\
\text { sodium dodecyl } \\
\text { sulfate }\end{array}$ & $\begin{array}{c}\text { n-Hexane } \\
\text { sodium dodecyl } \\
\text { sulfate }\end{array}$ & $\begin{array}{c}\text { n-Hexane } \\
\text { sodium dodecyl } \\
\text { sulfate }\end{array}$ \\
\hline $\mathrm{C}$ (additive $\mathrm{X} 1)$, wt $\%$ & 0.14 & 0.18 & 0.23 & 0.12 \\
\hline Polymerization time, $\mathrm{s}$ & 76 & 52 & 100 & 118 \\
\hline Heat curing time, min & 16 & 15 & 30 & 21 \\
\hline $\begin{array}{l}\text { Heat curing temperature, } \\
{ }^{\circ} \mathrm{C}\end{array}$ & 39 & 42 & 55 & 56 \\
\hline Substrate & PES, $0.2 \mu m$ & PES, $150 \mathrm{kDa}$ & PES, $150 \mathrm{kDa}$ & PES, $0.2 \mu \mathrm{m}$ \\
\hline TMP, bar & 2 & 2 & 2 & 2 \\
\hline Salt & $\mathrm{Na}_{2} \mathrm{SO}_{4}$ & $\mathrm{Na}_{2} \mathrm{SO}_{4}$ & $\mathrm{Na}_{2} \mathrm{SO}_{4}$ & $\mathrm{Na}_{2} \mathrm{SO}_{4}$ \\
\hline Salt concentration, $\mathrm{mg} \mathrm{L}^{-1}$ & 1000 & 1000 & 1000 & 1000 \\
\hline Membrane fabrication & \multicolumn{4}{|c|}{ Membrane } \\
\hline condition & \multicolumn{2}{|r|}{5} & \multicolumn{2}{|c|}{6} \\
\hline Material A1 & \multicolumn{2}{|c|}{ 1,2-diaminoprone } & \multicolumn{2}{|c|}{ 1-(2-aminoethyl)piperazine } \\
\hline Material A2 & \multicolumn{2}{|r|}{ I } & \multicolumn{2}{|c|}{ Diethanolamine } \\
\hline $\mathrm{A} 1 / \mathrm{A} 2$ ratio & \multicolumn{2}{|r|}{ I } & \multicolumn{2}{|c|}{4.55} \\
\hline
\end{tabular}




\begin{tabular}{|c|c|c|}
\hline $\mathrm{C}(\mathrm{A}), \mathrm{wt} \%$ & 0.98 & 0.65 \\
\hline Monomer B & TMC & TMC \\
\hline $\mathrm{C}(\mathrm{B}), \mathrm{wt} \%$ & 1.15 & 0.57 \\
\hline Organic solvent & n-Hexane & n-Hexane \\
\hline Additive $\mathrm{X} 1$ & sodium phosphate & l \\
\hline $\mathrm{C}$ (additive $\mathrm{X} 1$ ), wt $\%$ & 0.42 & l \\
\hline Nanomaterials type in & cellulose nanocrystal & cellulose nanocrystal \\
\hline aqueous phase & $(\mathrm{CNC})$ & $(\mathrm{CNC})$ \\
\hline $\mathrm{C}$ (nanomaterials-aqueous) & 0.016 & 0.025 \\
\hline nanomaterials morphology & rodlike & rodlike \\
\hline Polymerization time, $\mathrm{s}$ & 35 & 41 \\
\hline Heat curing time, min & 19 & 23 \\
\hline $\begin{array}{l}\text { Heat curing temperature, } \\
\qquad{ }^{\circ} \mathrm{C}\end{array}$ & 40 & 50 \\
\hline Substrate & PES, $150 \mathrm{kDa}$ & PES, $0.2 \mu \mathrm{m}$ \\
\hline TMP, bar & 3 & 3 \\
\hline Salt & $\mathrm{Na}_{2} \mathrm{SO}_{4}$ & $\mathrm{Na}_{2} \mathrm{SO}_{4}$ \\
\hline Salt Concentration, $\mathrm{mg} \mathrm{L}^{-1}$ & 1000 & 1000 \\
\hline
\end{tabular}


Table S7. Membrane materials and fabrication conditions used to fabricate membranes for validation of Bayesian optimization on water permeability and water $/ \mathrm{NaCl}$ selectivity.

\begin{tabular}{ccc}
\hline Membrane fabrication condition & Membrane & Membrane \\
Material A1 & $\mathbf{1}$ & $\mathbf{2}$ \\
Material A2 & Tri(2-aminoethyl)a-mine & 1-(2-Aminoethyl)piperazine \\
A1/A2 ratio & Polyethylenime, 1300 & $/$ \\
C (A), wt\% & 6.29 & $/$ \\
Monomer B & 1.14 & 0.87 \\
C (B), wt\% & TMC & TMC \\
Organic solvent & 0.78 & 0.58 \\
Additive X1 & n-Hexane & n-Hexane \\
C (additive X1), wt $\%$ & sodium phosphate & 0.15 \\
Polymerization time, $\mathrm{s}$ & 0.33 & 88 \\
Heat curing time, min & 35 & 14 \\
Heat curing temperature, ${ }^{\circ} \mathrm{C}$ & 10 & 50 \\
Substrate & 55 & PES, $0.2 \mu m$ \\
TMP, bar & PES, $150 \mathrm{kDa}$ & 2 \\
Salt & 2 & $\mathrm{NaCl}$ \\
Salt concentration, mg L ${ }^{-1}$ & $\mathrm{NaCl}$ & 1000 \\
\hline
\end{tabular}


Table S8. Predictive performance of different configurations of machine learning algorithms, encoder methods and scaler methods.

\begin{tabular}{|c|c|c|c|c|c|c|c|}
\hline Dataset & $\begin{array}{c}\text { RMSE } \\
\text { (training) }\end{array}$ & $\begin{array}{c}\mathbf{R}^{2} \\
\text { (training) }\end{array}$ & $\begin{array}{c}\text { RMSE } \\
\text { (test) }\end{array}$ & $\begin{array}{c}\mathbf{R}^{2} \\
\text { (test) }\end{array}$ & Regressor & Encoder & Scaler \\
\hline \multirow{5}{*}{$\begin{array}{c}\text { Water } \\
\text { permeability }\end{array}$} & 0.322010 & 0.996739 & 3.140497 & 0.681133 & XGBRegressor & $\begin{array}{c}\text { Backward } \\
\text { Difference } \\
\text { Encoder }\end{array}$ & $\begin{array}{c}\text { Power } \\
\text { Transformer }\end{array}$ \\
\hline & 0.627670 & 0.987626 & 3.247780 & 0.656712 & XGBRegressor & $\begin{array}{c}\text { Sum } \\
\text { Encoder }\end{array}$ & $\begin{array}{c}\text { Power } \\
\text { Transformer }\end{array}$ \\
\hline & 0.627670 & 0.987626 & 3.249084 & 0.656352 & XGBRegressor & $\begin{array}{l}\text { OneHot } \\
\text { Encoder }\end{array}$ & $\begin{array}{c}\text { Power } \\
\text { Transformer }\end{array}$ \\
\hline & 0.325847 & 0.996632 & 3.269983 & 0.651458 & XGBRegressor & $\begin{array}{c}\text { Backward } \\
\text { Difference } \\
\text { Encoder }\end{array}$ & $\begin{array}{l}\text { Standard } \\
\text { Scaler }\end{array}$ \\
\hline & 0.325847 & 0.996632 & 3.271000 & 0.651269 & XGBRegressor & $\begin{array}{l}\text { Backward } \\
\text { Difference } \\
\text { Encoder }\end{array}$ & $\begin{array}{l}\text { Robust } \\
\text { Scaler }\end{array}$ \\
\hline \multirow{5}{*}{$\begin{array}{c}\text { Salt } \\
\text { rejection }\end{array}$} & 3.562938 & 0.984872 & 11.577171 & 0.838997 & XGBRegressor & $\begin{array}{l}\text { Helmert } \\
\text { Encoder }\end{array}$ & $\begin{array}{l}\text { Robust } \\
\text { Scaler }\end{array}$ \\
\hline & 3.562938 & 0.984872 & 11.582038 & 0.838876 & XGBRegressor & $\begin{array}{l}\text { Helmert } \\
\text { Encoder }\end{array}$ & $\begin{array}{l}\text { MaxAbs } \\
\text { Scaler }\end{array}$ \\
\hline & 3.562938 & 0.984872 & 11.583294 & 0.838831 & XGBRegressor & $\begin{array}{l}\text { Helmert } \\
\text { Encoder }\end{array}$ & $\begin{array}{l}\text { Standard } \\
\text { Sclaler }\end{array}$ \\
\hline & 3.562938 & 0.984872 & 11.594645 & 0.838523 & XGBRegressor & $\begin{array}{l}\text { Helmert } \\
\text { Encoder }\end{array}$ & $\begin{array}{l}\text { MinMax } \\
\text { Scaler }\end{array}$ \\
\hline & 2.754982 & 0.990936 & 11.611519 & 0.837953 & XGBRegressor & $\begin{array}{l}\text { Binary } \\
\text { Encoder }\end{array}$ & $\begin{array}{c}\text { Power } \\
\text { Transformer }\end{array}$ \\
\hline
\end{tabular}

\section{REFERENCES}

1. Liang, Y.; Zhu, Y.; Liu, C.; Lee, K.-R.; Hung, W.-S.; Wang, Z.; Li, Y.; Elimelech, M.; Jin, J.; Lin, S., Polyamide nanofiltration membrane with highly uniform sub-nanometre pores for sub-1 $\AA$ precision separation. Nature communications 2020, 11, (1), 1-9.

2. Marcus, Y., Thermodynamics of solvation of ions. Part 5.-Gibbs free energy of hydration at 298.15 K. Journal of the Chemical Society, Faraday Transactions 1991, 87, (18), 2995-2999. 